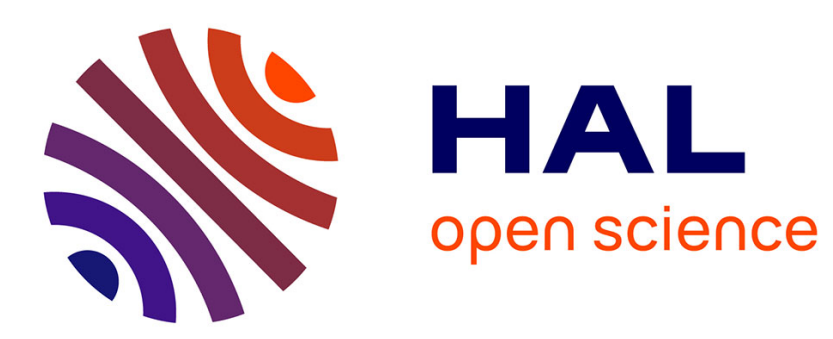

\title{
Multisource Discrimination Using IIR Volterra Filtering
}

Vincent Teuliere, André Monin, Gérard Salut

\section{To cite this version:}

Vincent Teuliere, André Monin, Gérard Salut. Multisource Discrimination Using IIR Volterra Filtering. IEEE Transactions on Communications, 2001, 49 (5), pp.922 - 931. 10.1109/26.923815. hal-02915718

\section{HAL Id: hal-02915718 \\ https://hal.laas.fr/hal-02915718}

Submitted on 17 Aug 2020

HAL is a multi-disciplinary open access archive for the deposit and dissemination of scientific research documents, whether they are published or not. The documents may come from teaching and research institutions in France or abroad, or from public or private research centers.
L'archive ouverte pluridisciplinaire HAL, est destinée au dépôt et à la diffusion de documents scientifiques de niveau recherche, publiés ou non, émanant des établissements d'enseignement et de recherche français ou étrangers, des laboratoires publics ou privés. 


\title{
Multi-emission discrimination using IIR Volterra Filtering
}

\author{
André MONIN, Gérard SALUT,Vincent TEULIERE. \\ Laboratoire d'Analyse et d'Architecture des Systèmes. \\ Centre National de la Recherche Scientifique. \\ 7 Avenue du Colonel Roche 31077 TOULOUSE Cedex. FRANCE \\ Tel: (33).05.61.33.62.95 / Fax: (33).05.61.55.69.69 / email: monin@laas..fr
}

\section{Abstract:}

Keywords: .

\section{Introduction.}

The aim of this paper is to build a test algorithm devoted to detect when several independent emissions are using the same carrier and, in such a situation, to determine the number of them.

Let us consider the case of phasis or frequency modulation. If there is only one modulation in the observed signal, its module remains constant with respect to time and the fluctuations of the module of the observed signal is only due to additive white noise. If now, one consider the sum of two modulations, as the phasis of each modulations are assumed to be independents, the module of the observed signal will be fluctuating. As a consequence of this remark, the algorithm which is proposed is based on the prediction of the module of the observed signal conditionally to the number of modulation supposed. The innovations involved are then used to construct a sufficient statistic for the detection in the sense that its expectation maximize some contrast criterium.

\section{Statistics of a multi-emission.}

In our problem, the hypothesis to be tested is the number $n$ of emissions in the observed complex signal $y_{t}$ obtain after two channels demodulation. It can be written:

$$
y_{t}=s_{t}^{n}+v_{t}
$$

where:

$$
s_{t}^{n}=\sum_{i=1}^{n} x_{t}^{i}
$$

is the sum of $n$ independent modulations and $v_{t}=v_{t}^{1}+j v_{t}^{2}$ is a complex white noise with real and imaginary assumed to be independent and of variance $R$. The moments of the white noise $v_{t}$ are all zero except the following:

$$
E\left[\left|v_{t}\right|^{2 k}\right]=R^{k} \sum_{p=0}^{k} C_{k}^{p} \alpha_{p} \alpha_{k-p}
$$

with: 


$$
C_{k}^{p}=\frac{k !}{p !(k-p) !} \text { and } \alpha_{p}=1 \times 3 \times \ldots \times(2 p-1)
$$

Let us examine the statistics of multi-emission in the particular case of a digital phasis modulation. Each signal can be represented, after demodulation, as the complex signal:

$$
x_{t}=x_{0} \exp \left(j \varphi_{t}\right)
$$

with $x_{0}=A_{0} \exp \left(j \varphi_{0}\right)$ where $A_{0}$ is the (constant) amplitude of the signal and $\varphi_{0}$ the initial phasis. $\varphi_{t}$ is the current phasis carrying the information to be send. Let $\Delta t$ be the sample period. Between to samples, the evolution of the signal is defined as:

$$
x_{t+\Delta t}=x_{t} \exp \left(j \Delta \varphi_{t}\right)
$$

where $\Delta \varphi_{t}$ designates the phasis increment. As the transmitted information is unknown, $\Delta \varphi_{t}$ may be viewed as a white noise with characteristics depending of the kind of modulation used. Let $w_{t}=\exp \left(j \Delta \varphi_{t}\right)$.

For an analogical modulation, the density of probability of $\Delta \varphi_{t}$ may be assumed gaussian. In such a situation, if the variance of $\Delta \varphi_{t}$ is $Q$, the moments of $w_{t}$ are defined by:

$$
\begin{aligned}
& \forall k \neq l, E\left[w_{t}^{k}\left(w_{t}^{*}\right)^{l}\right]=\exp \left(-\frac{1}{2}(k-l)^{2} Q\right) \\
& \forall k, E\left[w_{t}^{k}\left(w_{t}^{*}\right)^{k}\right]=1
\end{aligned}
$$

where $w^{*}$ is complex conjugate of $w$.

For a digital modulation, the phasis jumps time of occurrence may be modelised with a Poisson process of frequency $\lambda$ and the amplitude uniformly distributed on $m$ points on the unit circle. In that case, the density of probability of $\Delta \varphi_{t}$ is:

$$
p\left(\Delta \varphi_{t}\right)=(1-\lambda \Delta t) \delta_{0}\left(\Delta \varphi_{t}\right)+\frac{\lambda \Delta t}{m-1} \sum_{p=1}^{m-1} \delta_{\frac{2 \pi p}{m}}\left(\Delta \varphi_{t}\right)
$$

where $\delta_{a}(\Delta \varphi)$ is the Dirac distribution centered on $a$. The resulting moments of $w_{t}$ are then defined by:

$$
\begin{aligned}
& \forall k \neq l, E\left[w_{t}^{k}\left(w_{t}^{*}\right)^{l}\right]=1-\frac{m}{m-1} \lambda \Delta t \\
& \forall k, E\left[w_{t}^{k}\left(w_{t}^{*}\right)^{k}\right]=1
\end{aligned}
$$

One may of course consider other kind of modulations but it is important to note that, for all types of modulations, one has always the same property:

$$
\begin{array}{r}
\forall k \neq l, E\left[w_{t}^{k}\left(w_{t}^{*}\right)^{l}\right]=a \\
\forall k, E\left[w_{t}^{k}\left(w_{t}^{*}\right)^{k}\right]=1
\end{array}
$$

with $a<1$.

Let us now analyze the evolution of moments of one modulation. According to (2.10), one has:

$$
\begin{aligned}
\forall k \neq l, E\left[x_{t+\Delta t}^{k}\left(x_{t+\Delta t}^{*}\right)^{l}\right] & =a E\left[x_{t}^{k}\left(x_{t}^{*}\right)^{l}\right] \\
\forall k, E\left[x_{t+\Delta t}^{k}\left(x_{t+\Delta t}^{*}\right)^{k}\right] & =E\left[x_{t}^{k}\left(x_{t}^{*}\right)^{k}\right]
\end{aligned}
$$

As a consequence, because $a<1$, it is clear that all moments of $x_{t}$ are asymptotically zero expect the set $\left\{E\left[x_{t}^{k}\left(x_{t}^{*}\right)^{k}\right], k \geq 1\right\}$ which are constant. 
The first moment of a multi-emission can now be computed using (2.11). One may derive that all moments of degree $\leq 4$ are asymptotically zero except the following:

$$
\begin{gathered}
E\left[s_{t}^{n} s_{t}^{n^{*}}\right]=\sum_{k=1}^{n} E\left[x_{t}^{k} x_{t}^{k}\right] \\
E\left[\left(s_{t}^{n} s_{t}^{n^{*}}\right)^{2}\right]=\sum_{k=1}^{n} E\left[\left(x_{t}^{k} x_{t}^{k^{*}}\right)^{2}\right]+2 \sum_{\substack{k \neq l \\
k, l=1}}^{n} E\left[x_{t}^{k} x_{t}^{k^{*}}\right] E\left[x_{t}^{l} x_{t}^{l^{*}}\right]
\end{gathered}
$$

Let us compare these moments when the global power $P$ is the same but when the number of modulation is different. On has:

$$
\forall k, E\left[x_{t}^{k} x_{t}^{k}\right]=P / n
$$

and the fourth order moment is:

$$
E\left[\left(s_{t}^{n} s_{t}^{n}{ }^{*}\right)^{2}\right]=\frac{1+2(n-1)}{n}\left(E\left[s_{t}^{n} s_{t}^{n^{*}}\right]\right)^{2}
$$

This relation means that the number of signals is detectable as the ratio within the fourth order moment and the square of the second order moment depends on $n$. This property will be exploited in the sequel to construct the detection test based on predictors of the signal square module.

\section{The IIR Volterra quadratic predictor.}

Let us applied the quadratic Volterra predictor as defined in [2] to our problem. When one deals with real numbers, the purely quadratic IIR predictor of any function $\phi_{t}$ of the state has the following recursive structure:

$$
\begin{aligned}
p_{t+\Delta t} & =A_{t}^{1} p_{t}+A_{t}^{0} y_{t} \\
z_{t+\Delta t} & =A_{t}^{2} z_{t}+p_{t+\Delta t} y_{t}
\end{aligned}
$$

where $z_{t}$ is the output and $\left\{A_{t}^{i}\right\}$ are time variant parameters computed to minimize the mean square prediction error, that is:

$$
J_{t}=\frac{1}{2} E\left[\left\|\phi_{t}-z_{t}\right\|^{2}\right]
$$

The optimal parameters are computed using the following orthogonalities:

$$
E\left[\left(\phi_{t+\Delta t}-z_{t+\Delta t}\right)\left[\begin{array}{c}
y_{t}^{2} \\
p_{t}^{T} y_{t} \\
z_{t}^{T}
\end{array}\right]\right]=0
$$

which leads to the following linear system:

$$
\begin{aligned}
A_{t}^{2} E\left[z_{t}\left(y_{t}\right)^{2}\right]+A_{t}^{1} E\left[p_{t}\left(y_{t}\right)^{3}\right]+A_{t}^{0} E\left[\left(y_{t}\right)^{4}\right] & =E\left[\phi_{t+\Delta t}\left(y_{t}\right)^{2}\right] \\
A_{t}^{2} E\left[z_{t} p_{t}^{T} y_{t}\right]+A_{t}^{1} E\left[z_{t}^{1} p_{t}^{T}\left(y_{t}\right)^{2}\right]+A_{t}^{0} E\left[p_{t}^{T}\left(y_{t}\right)^{3}\right] & =E\left[\phi_{t+\Delta t} p_{t}^{T} y_{t}\right] \\
A_{t}^{2} E\left[z_{t} z_{t}^{T}\right]+A_{t}^{1} E\left[p_{t} z_{t}^{T}\left(y_{t}\right)^{2}\right]+A_{t}^{0} E\left[z_{t}^{T}\left(y_{t}\right)^{2}\right] & =E\left[\phi_{t+\Delta t} z_{t}^{T}\right]
\end{aligned}
$$


The optimal parameters are then computable if all the expectations in (3.4) are computable. Note that this condition is achieved when the prediction system of $\phi_{t}$ is bilinear.

Let us generalize this estimator to the complex number case. Let $\phi_{t}=\left|s_{t}^{n}\right|^{2}$ to be estimated. It is easy to verify that, according to (2.13), the only non-zero quadratic projection of $\phi_{t}$ is the projection over the term $y_{t} y_{t}{ }^{*}$. As a consequence, the structure of the predictor must be as following:

$$
\begin{aligned}
p_{t+\Delta t} & =A_{t}^{1} p_{t}+A_{t}^{0} y_{t} \\
z_{t+\Delta t} & =A_{t}^{2} z_{t}+p_{t+\Delta t} y_{t}^{*}+p_{t+\Delta t}^{*} y_{t}+B_{t}
\end{aligned}
$$

with $z_{t} \in R$. The term $B_{t}$ makes sure that the predictor is not biased.

The stochastic observability concept introduced in [2] is used here to determine the dimension of the predictor, that is the dimension of $z_{t}$. In the general case, the variable $\Pi_{t}+\Delta t^{\dagger}$ is stochastically observable if the following relation:

$$
\Pi_{t+\Delta t} \rightarrow\left\{E\left(\left[y_{\tau_{1}} y_{\tau_{2}}^{*} \mid \Pi_{t+\Delta t}\right], \forall \tau_{1}, \tau_{2} \leq t\right)\right\}
$$

is one to one. To verify this property, let us compute first the conditional expectation:

$$
\begin{aligned}
E\left[y_{t} y_{t}^{*} \mid \Pi_{t+\Delta t}\right] & =\sum_{k, l=1}^{n} E\left[x_{t}^{k} x_{t}^{l^{*}} \mid \Pi_{t+\Delta t}\right]+2 R \\
& =\sum_{k=1}^{n} E\left[x_{t}^{k} x_{t}^{k} \mid \Pi_{t+\Delta t}\right]+\sum_{k \neq l}^{n} E\left[x_{t}^{k} x_{t}^{l^{*}} \mid \Pi_{t+\Delta t}\right]+2 R
\end{aligned}
$$

According to (2.11), one may write:

$E\left[y_{t} y_{t}^{*} \mid \Pi_{t+\Delta t}\right]=\sum_{k=1}^{n} E\left[x_{t+\Delta t}^{k} x_{t+\Delta t^{\dagger}}^{k^{*}} \mid \Pi_{t+\Delta t}\right]+\frac{1}{a} \sum_{k \neq l}^{n} E\left[x_{t+\Delta t}^{k} x_{t+\Delta t^{\dagger}}^{l^{*}} \mid \Pi_{t+\Delta t}\right]+2 R$

In the same way, one has:

$E\left[y_{t-\Delta t} y_{t-\Delta t}^{*} \mid \Pi_{t+\Delta t}\right]=\sum_{k=1}^{n} E\left[x_{t+\Delta t}^{k} x_{t+\Delta t^{\dagger}}^{k^{*}} \mid \Pi_{t+\Delta t}\right]+\frac{1}{a^{2}} \sum_{k \neq l}^{n} E\left[x_{t+\Delta t}^{k} x_{t+\Delta t^{\dagger}}^{l^{*}} \mid \Pi_{t+\Delta t}\right]+2 R$

Let:

$$
\Pi_{t}=\left[\sum_{k=1}^{n} x_{t}^{k} x_{t}^{k^{*}}, \sum_{k \neq l}^{n} x_{t}^{k} x_{t}^{l^{*}}\right]
$$

It is clear that the relation $\Pi_{t+\Delta t} \rightarrow\left\{E\left[y_{t} y_{t}^{*} \mid \Pi_{t+\Delta t}\right], E\left[y_{t-\Delta t} y_{t-\Delta t}^{*} \mid \Pi_{t+\Delta t}\right]\right\}$ is one to one. As a consequence, the dimension of $z_{t}$ must be at least two. Note that it is equivalent to consider $\Pi_{t}$ and the more natural following set:

$$
\Psi_{t}=\left[\sum_{k=1}^{n} x_{t}^{k} x_{t}^{k^{*}},\left|\sum_{k=1}^{n} x_{t}^{k}\right|^{2}\right]
$$

Note moreover that if $n=1$, the two components of $\Psi_{t}$ are identical and the dimension of the quadratic predictor is reduced to 1 . 


\section{Detection test.}

The detection test used here takes one's inspiration from the linear gaussian case. Recall that in that classical case, one constructs the optimal predictor $\hat{x}_{(k+1) \Delta t \mid k \Delta t}^{n}$ of the signal under each hypothesis $H_{n}$. One then computes the likelihood of each hypothesis $H_{n}$ that has the following expression:

$$
L_{t}^{n}=\sum_{k=0}^{t / \Delta t} \frac{\left(y_{(k+1) \Delta t}-\hat{x}^{n}{ }_{(k+1) \Delta t \mid k \Delta t}\right)^{2}}{E\left[\left(y_{(k+1) \Delta t}-\hat{x}^{n}(k+1) \Delta t \mid k \Delta t\right)^{2}\right]}+\log \left(E\left[\left(y_{(k+1) \Delta t}-\hat{x}^{n}{ }_{(k+1) \Delta t \mid k \Delta t}\right)^{2}\right] \ell^{2}\right.
$$

The decision process is then defined as: if $L_{t}^{n}<L_{t}^{m_{2}}$, the hypothesis $n$ is chosen.

Our test is based on the innovation processes generated by the signal square module prediction. Let $\hat{\phi}^{n}{ }_{t+\Delta t \mid t}$ be the one step predictor of $\phi_{t}$ under hypothesis " $n$ modulations in the signal". The test variable $S_{t}$ is imposed to have the following form:

$$
S_{t}=\sum_{k=0}^{t / \Delta t}\left(\left|y_{(k+1) \Delta t}\right|^{2}-\hat{\phi}^{n_{1}}(k+1) \Delta t \mid k \Delta t\right)^{2}+\alpha_{k}\left(\left|y_{(k+1) \Delta t}\right|^{2}-\hat{\phi}^{n_{2}}(k+1) \Delta t \mid k \Delta t\right)^{2}+\beta_{k}
$$

where $\alpha_{k}$ and $\beta_{k}$ are parameters devoted to normalize the contrast within the two hypothesis. A natural way to satisfied this condition is to impose:

$$
\begin{aligned}
& E\left[S_{t} \mid H_{n_{1}}\right]=t \\
& E\left[S_{t} \mid H_{n_{2}}\right]=-t
\end{aligned}
$$

assuring that the expectation of the distance within the test variable under each hypothesis increases linearly with the number of data treated. The test is then reduced to:

$$
S_{t}<0 \rightarrow n_{1} \text { and } S_{t}>0 \rightarrow n_{2}
$$

The constraints (4.3) leads to the following values of $\alpha_{k}, \beta_{k}$ :

$$
\begin{aligned}
& \alpha_{k}=\frac{2-J_{k}^{23}+J_{k}^{22}}{J_{k}^{33}-J_{k}^{32}} \\
& \beta_{k}=1-J_{k}^{23}-\alpha_{k} J_{k}^{33}
\end{aligned}
$$

where:

$$
J_{k}^{i j}=E\left[\left(\left|y_{(k+1) \Delta t}\right|^{2}-\hat{\phi}^{n_{i}}(k+1) \Delta t \mid k \Delta t\right)^{2} \mid H_{n_{j}}\right]
$$

Note that all the variances $J_{k}^{i j}$ may be computed a priori since the system is bilinear.

\section{Simulation results.}

In all the following simulations. the signal to noise ratio is fixed to $10 \mathrm{~dB}$. 


\subsection{Detection within 1 and 2 modulations.}

The first step of construction of the test is the computation of the predictor of the signal square module. One shows on figure 1 the estimation of the signal square module under each hypothesis when only one signal is present with power equal one. Il appears clearly that the estimator which deals with $H_{1}$, the good hypothesis, is much more accurate than the estimator which deals with $\mathrm{H}_{2}$. The situation is inverted when two signals are present as it appears on figure 2.
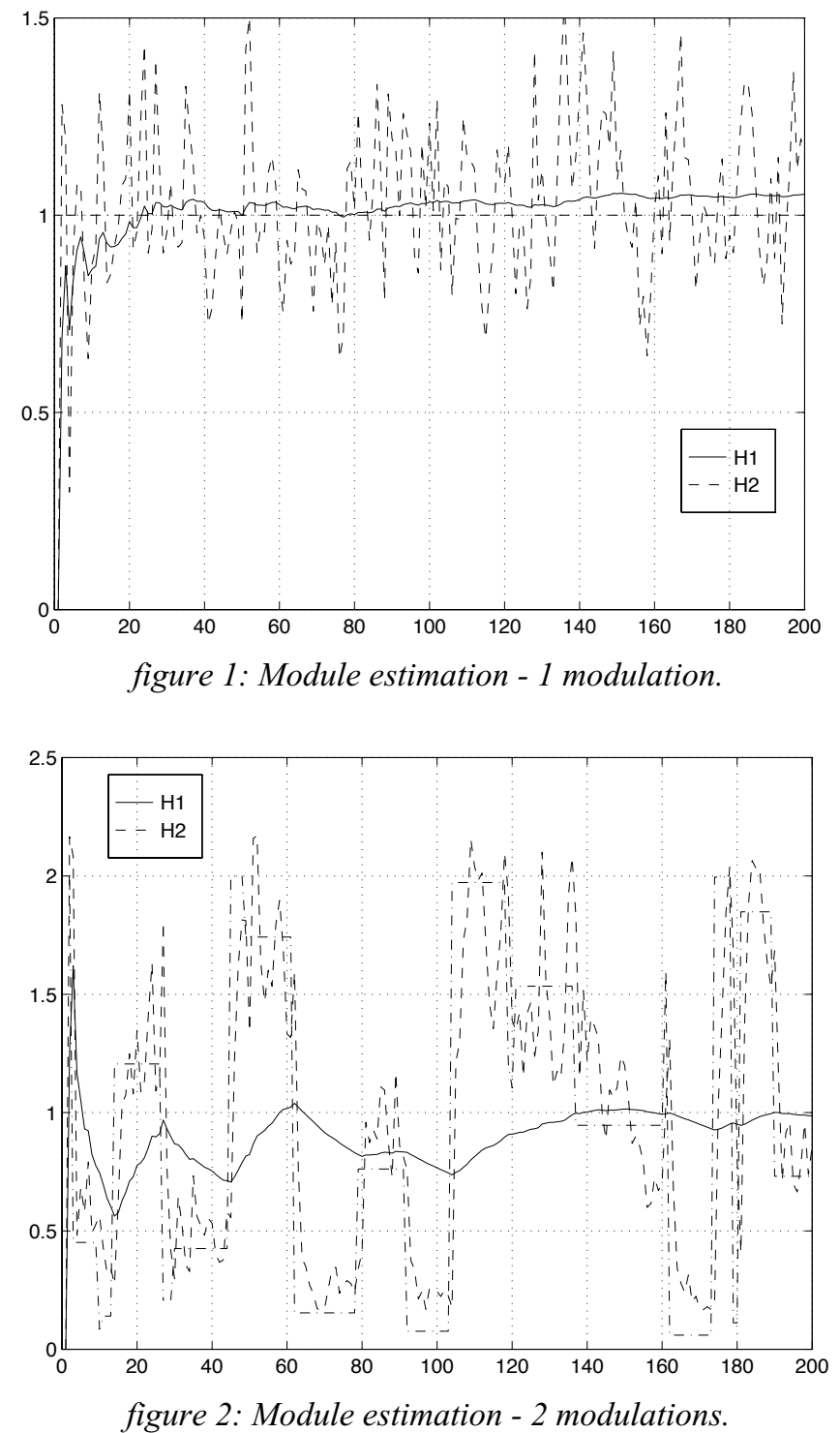

The test variable $S_{t}$ as defined by (4.2) is presented on figure 3 . The dotted lines are the expectations of $S_{t}$ under each hypothesis. One may oberve that the detection is very fast. 


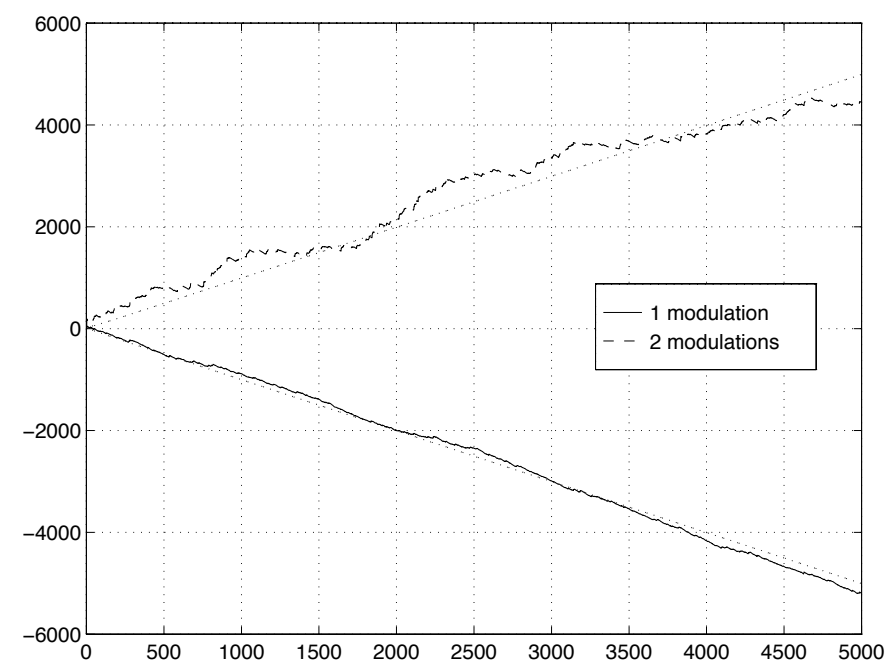

figure 3: Detection within 1 and 2 modulations.

\subsection{Detection within 2 and 3 modulations.}

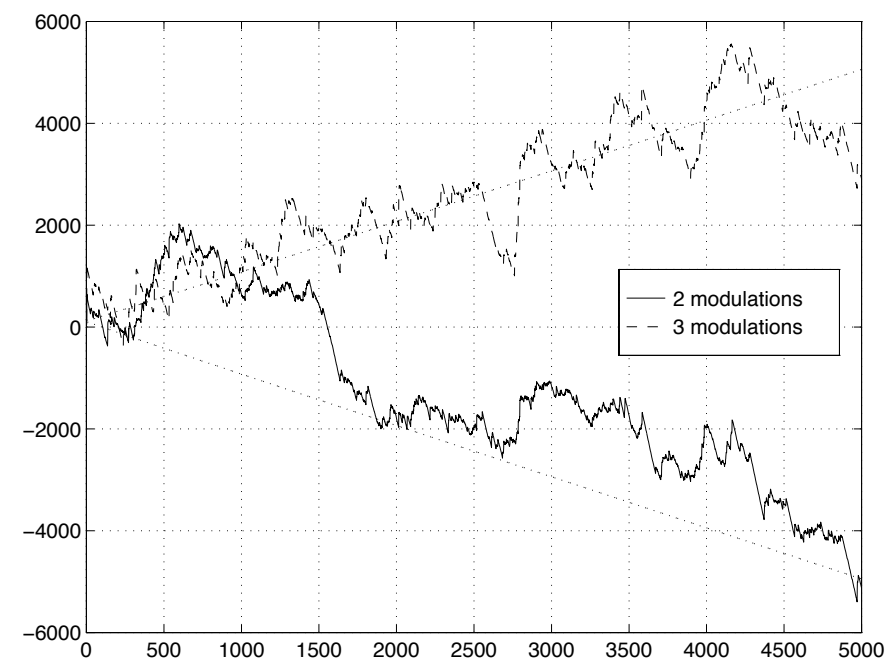

figure 4: Dtection within 2 and 3 modulations

\section{References.}

[1] H. L. Van Trees

[2] A. Monin, G. Salut, IIR Volterra Filtering With Application to Bilinear Systems. IEEE Trans. on Signal Processing, vol. 44, nº, pp. 2209-2221, 1996. 